\title{
THE SMALLEST POSITIVE EIGENVALUE OF A QUASISYMMETRIC AUTOMORPHISM OF THE UNIT CIRCLE
}

\author{
DARIUSZ PARTYKA \\ Department of Mathematics, Maria Curie-Sktodowska University \\ Pl. M. Curie-Sktodowskiej 1, 20-031 Lublin, Poland \\ E-mail: partyka@plumcs11.bitnet
}

\begin{abstract}
This paper provides sufficient conditions on a quasisymmetric automorphism $\gamma$ of the unit circle which guarantee the existence of the smallest positive eigenvalue of $\gamma$. They are expressed by means of a regular quasiconformal Teichmüller self-mapping $\varphi$ of the unit disc $\Delta$. In particular, the norm of the generalized harmonic conjugation operator $A_{\gamma}: \mathbb{H} \rightarrow$ $\mathbb{H}$ is determined by the maximal dilatation of $\varphi$. A characterization of all eigenvalues of a quasisymmetric automorphism $\gamma$ in terms of the smallest positive eigenvalue of some other quasisymmetric automorphism $\sigma$ is given.
\end{abstract}

1. Introduction. Let us denote by $\mathbb{Q}_{\mathbf{T}}(K), 1 \leq K<\infty$, the class of all homeomorphic self-mappings of the unit circle $\mathbf{T}=\{z \in \mathbb{C}:|z|=1\}$ which admit a $K$-quasiconformal extension to the unit disc $\Delta=\{z \in \mathbb{C}:|z|<1\}$ and let $\mathbb{Q}_{\mathbf{T}}=\bigcup_{1<K<\infty} \mathbb{Q}_{\mathbf{T}}(K)$. For any homeomorphism $\gamma \in \mathbb{Q}_{\mathbf{T}}$ we set $K(\gamma)=\inf \{K$ : $\left.\gamma \in \mathbb{Q}_{\mathbf{T}}(K)\right\}$. Due to J. G. Krzyż, cf. $[\mathrm{K}]$, the class $\mathbb{Q}_{\mathbf{T}}$ coincides with the class of all quasisymmetric automorphisms of the unit circle $\mathbf{T}$, i.e. all sense-preserving homeomorphisms $\gamma: \mathbf{T} \rightarrow \mathbf{T}$ satisfying

$$
k^{-1} \leq\left|\gamma\left(I_{1}\right)\right| /\left|\gamma\left(I_{2}\right)\right| \leq k
$$

for each pair of adjacent closed $\operatorname{arcs} I_{1}, I_{2} \subset \mathbf{T}$ of equal length: $0<\left|I_{1}\right|=$ $\left|I_{2}\right| \leq \pi$ where the constant $k$ depends on $\gamma$ only. Let us denote by $L_{\mathbf{T}}^{p}, 1 \leq$ $p<\infty$, the space of all functions $f: \mathbf{T} \rightarrow \mathbb{R}, p$-integrable on $\mathbf{T}$, i.e. $\|f\|_{p}=$

1991 Mathematics Subject Classification: 30C62, 45C05.

Key words and phrases: quasiconformal mappings, Teichmüller mappings, harmonic conjugation operator, eigenvalues and spectral values of a linear operator, quasisymmetric automorphisms.

Research supported in part by KBN Grant \#PB2-11-70-9101.

The paper is in final form and no version of it will be published elsewhere. 
$\left(\int_{\mathbf{T}}|f(z)|^{p}|d z|\right)^{1 / p}<\infty$ and let $L_{\mathbf{T}}^{\infty}=\left\{f \in L_{\mathbf{T}}^{1}:\|f\|_{\infty}=\operatorname{sup~ess}_{z \in \mathbf{T}}|f(z)|<\infty\right\}$. The space $L_{\mathbf{T}}^{2}$ is a real Hilbert space with the inner product

$$
(f, g)=\int_{\mathbf{T}} f(z) g(z)|d z|, \quad f, g \in L_{\mathbf{T}}^{2} .
$$

With any function $f \in L_{\mathbf{T}}^{1}$ we can associate an analytic function $f_{\Delta}: \Delta \rightarrow \mathbb{C}$ given by the formula

$$
\begin{aligned}
f_{\Delta}(z) & =\frac{1}{2 \pi} \int_{\mathbf{T}} f(u) \frac{u+z}{u-z}|d u| \\
& =\frac{1}{2 \pi} \int_{\mathbf{T}} f(u)|d u|+\frac{1}{\pi} \sum_{n=1}^{\infty}\left(\int_{\mathbf{T}} f(u) \bar{u}^{n}|d u|\right) z^{n}, \quad z \in \Delta .
\end{aligned}
$$

The space $\mathbb{H}=\left\{f \in L_{\mathbf{T}}^{1}: \int_{\Delta}\left|f_{\Delta}^{\prime}\right|^{2} d S<\infty\right.$ and $\left.f_{\Delta}(0)=0\right\}$, where $f_{\Delta}^{\prime}=\left(f_{\Delta}\right)^{\prime}$, equipped with the inner product $(f, g)_{\mathbb{H}}=\operatorname{Re} \int_{\Delta} f_{\Delta}^{\prime} \overline{g_{\Delta}^{\prime}} d S, f, g \in \mathbb{H}$, is a real Hilbert space, isometric with the space $\widetilde{L}_{\mathbf{T}}^{2}=\left\{f \in L_{\mathbf{T}}^{2}: f_{\Delta}(0)=0\right\}$, cf. [P1, Theorem 1.2]. In the paper [P2] a linear homeomorphism $A_{\gamma}$ of the Hilbert space $\mathbb{H}$ onto itself was associated with every quasisymmetric automorphism $\gamma \in \mathbb{Q}_{\mathbf{T}}$. If $\gamma \in \mathbb{Q}_{\mathbf{T}}$ is sufficiently regular then the operator $A_{\gamma}$ has a nice form, cf. [P2, Theorem 1.4], given by means of a singular integral

$$
A_{\gamma}(f)(z)=\frac{1}{\pi} \text { ReP.V. } \int_{\mathbf{T}} \frac{f(u)}{\gamma(z)-\gamma(u)} d \gamma(u)-a_{\gamma}(f)
$$

for a.e. $z \in \mathbf{T}$ and $f \in \mathbb{H}$ where

$$
a_{\gamma}(f)=\frac{1}{2 \pi} \int_{\mathbf{T}}\left(\frac{1}{\pi} \operatorname{Re} \text { P.V. } \int_{\mathbf{T}} \frac{f(u)}{\gamma(z)-\gamma(u)} d \gamma(u)\right)|d z|
$$

is a normalization constant. If $\gamma(z)=z, z \in \mathbf{T}$, then $A_{\gamma}$ becomes the usual harmonic conjugation operator $A$, see $[\mathrm{G}]$. Moreover, $A_{\gamma}^{2}=-I$ for any $\gamma \in \mathbb{Q}_{\mathbf{T}}$, where $I$ is the identity operator. Thus $A_{\gamma}$ may be called a generalized harmonic conjugation operator. For basic properties of the operator $A_{\gamma}$ we refer to [P2]. Now we quote two properties essential for our considerations. Namely, $A A_{\gamma}: \mathbb{H} \rightarrow \mathbb{H}$ is a symmetric operator and

$$
A_{\gamma}=B_{\gamma} A B_{\gamma^{-1}}
$$

where $B_{\gamma}$ is a linear homeomorphism of the space $\left(\mathbb{H},\|\cdot\|_{\mathbb{H}}\right)$ onto itself such that

$$
B_{\gamma}(f)=f \circ \gamma-(f \circ \gamma)_{\Delta}(0) \text { on } \mathbf{T}
$$

for every continuous function $f \in \mathbb{H}$. This shows that the operator $A_{\gamma}$ is related to the Neumann-Poincaré integral operator of a Jordan curve $\Gamma$. More precisely, eigenvalues of the Neumann-Poincaré kernel $k$, cf. [BS], [S], correspond to eigenvalues of the symmetric operator $A A_{\gamma}: \mathbb{H} \rightarrow \mathbb{H}$ where $\gamma$ is a welding homeomorphism of a sufficiently smooth Jordan curve $\Gamma$, cf. $[\mathrm{P} 1],[\mathrm{KP}]$. This justifies 
introducing the notion of an eigenvalue and a spectral value of a quasisymmetric automorphism of the unit circle, by means of the spectrum of the operator $A A_{\gamma}$, cf. [P3], or equivalently, by means of the spectrum of the operator $R_{\gamma}$, cf. [P1], $[\mathrm{KP}]$, because $R_{\gamma}=I+A A_{\gamma}$, cf. [P2, (2.4)]. For the reader's convenience we quote

Definition 1.1. A real number $\lambda$ is said to be an eigenvalue of a quasisymmetric automorphism $\gamma \in \mathbb{Q}_{\mathbf{T}}$ if there exists a function $f \in \mathbb{H}$ with the norm $\|f\|_{\mathbb{H}}=1$ such that

$$
(\lambda+1) A(f)=(\lambda-1) A_{\gamma}(f) .
$$

The function $f$ is said to be an eigenfunction of $\gamma$ associated with the eigenvalue $\lambda$.

The set of all eigenvalues of $\gamma \in \mathbb{Q}_{\mathbf{T}}$ is denoted by $\Lambda_{\gamma}^{*}$.

DeFinition 1.2. A real number $\lambda$ is said to be a spectral value or an approximate eigenvalue of a quasisymmetric automorphism $\gamma \in \mathbb{Q}_{\mathbf{T}}$ if there exist functions $f_{n} \in \mathbb{H},\left\|f_{n}\right\|_{\mathbb{H}}=1, n=1,2, \ldots$ such that

$$
\left\|(\lambda+1) A\left(f_{n}\right)-(\lambda-1) A_{\gamma}\left(f_{n}\right)\right\|_{\mathbb{H}} \rightarrow 0 \quad \text { as } \quad n \rightarrow \infty .
$$

The set of all spectral values of $\gamma \in \mathbb{Q}_{\mathbf{T}}$ is denoted by $\Lambda_{\gamma}$. From [P2, Theorem 2.2] we are able to infer the following basic properties of the spectra $\Lambda_{\gamma}^{*}$ and $\Lambda_{\gamma}$ :

(i) $\Lambda_{\gamma}=\emptyset$ iff $\gamma=\mathbb{Q}_{\mathbf{T}}(1)$;

(ii) $\Lambda_{\gamma}^{*} \subset \Lambda_{\gamma}$;

(iii) if $\lambda \in \Lambda_{\gamma}$ then $|\lambda| \geq \frac{K(\gamma)+1}{K(\gamma)-1}$;

(iv) for every $\nu, \eta \in \mathbb{Q}_{\mathbf{T}}(1) \Lambda_{\gamma}=\Lambda_{\nu \circ \gamma \circ \eta}$ and $\Lambda_{\gamma}^{*}=\Lambda_{\nu \circ \gamma \circ \eta}^{*}$;

(v) $\Lambda_{\gamma}=\Lambda_{\gamma^{-1}}=\Lambda_{\bar{i}_{\mathbf{T}} \circ \gamma \circ \bar{i}_{\mathbf{T}}}$ and $\Lambda_{\gamma}^{*}=\Lambda_{\gamma^{-1}}^{*}=\Lambda_{\bar{i}_{\mathbf{T}} \circ \gamma \circ \bar{i}_{\mathbf{T}}}^{*}$;

(vi) if $\lambda \in \Lambda_{\gamma}$ then $-\lambda \in \Lambda_{\gamma}$ and if $\lambda \in \Lambda_{\gamma}^{*}$ then $-\lambda \in \Lambda_{\gamma}^{*}$

where $i_{\mathbf{T}}(z)=z, z \in \mathbf{T}$. For the proof of these properties cf. [P3]. A natural question appears when is the inequality (iii) sharp, i.e. when

$$
\inf \left\{|\lambda|: \lambda \in \Lambda_{\gamma}\right\}=\frac{K(\gamma)+1}{K(\gamma)-1}
$$

This is strictly related to the problem of determining the norm $\left\|A_{\gamma}\right\|$ which always does not exceed $K(\gamma)$, cf. [P2, Theorem 1.3]. Namely, $\left\|A_{\gamma}\right\|=K(\gamma)$ iff the equality (1.5) holds. In Section 2 of this paper we establish Theorem 2.2 giving a sufficient condition on a quasisymmetric automorphism $\gamma \in \mathbb{Q}_{\mathbf{T}}$ which guarantees the existence of the smallest positive eigenvalue $\lambda \in \Lambda_{\gamma}^{*}$ satisfying (1.5). In particular, this implies the equality $\left\|A_{\gamma}\right\|=K(\gamma)$. As a consequence we obtain Corollary 2.3 which characterizes every positive eigenvalue $\lambda \in \Lambda_{\gamma}^{*}$ as the smallest positive eigenvalue of some other quasisymmetric automorphism $\sigma \in \mathbb{Q}_{\mathbf{T}}$. 
2. Main results and proofs. In what follows we need the following

LEMMA 2.1 If $\varphi \in \mathbb{Q}_{\Delta}$ is a quasiconformal extension of a quasisymmetric automorphism $\gamma \in \mathbb{Q}_{\mathbf{T}}$ and there exist functions $f, g \in \mathbb{H}$ and a constant $c \in \mathbb{C}$ satisfying the equality

$$
\operatorname{Re} g_{\Delta}(z)=\operatorname{Re} f_{\Delta} \circ \varphi(z)+c, \quad z \in \Delta,
$$

then $g=B_{\gamma}(f)$.

Proof. By $\left[\mathrm{P} 1\right.$, Theorem 1.2] $C_{\mathbf{T}} \cap \mathbb{H}$ is a dense subset of the space $\left(\mathbb{H},\|\cdot\|_{\mathbb{H}}\right)$ where the class $C_{\mathbf{T}}$ consists of all continuous real-valued functions on the unit circle $\mathbf{T}$. Thus there exist functions $f_{n}, h_{n} \in C_{\mathbf{T}} \cap \mathbb{H}, n \in \mathbb{N}$, approximating the functions $f, g$ in $\left(\mathbb{H},\|\cdot\|_{\mathbb{H}}\right)$, respectively, i.e.

$$
\left\|f_{n}-f\right\|_{\mathbb{H}} \rightarrow 0 \quad \text { and } \quad\left\|h_{n}-g\right\|_{\mathbb{H}} \rightarrow 0 \quad \text { as } n \rightarrow \infty .
$$

Since a harmonic function minimizes the Dirichlet integral within the class of real continuous functions on the closed unit disc $\bar{\Delta}$ with given boundary values and absolutely continuous on a.e. chord of $\bar{\Delta}$, parallel to the coordinate axes, setting $g_{n}=B_{\gamma}\left(f_{n}\right)=f_{n} \circ \gamma-\left(f_{n} \circ \gamma\right)_{\Delta}(0), n \in \mathbb{N}$, we obtain by $(2.1)$ that for any $n \in \mathbb{N}$

$$
\int_{\Delta}\left|\left(g_{n}\right)_{\Delta}^{\prime}-g_{\Delta}^{\prime}\right|^{2} d S
$$

$$
\begin{aligned}
& =\lim _{m \rightarrow \infty} \int_{\Delta}\left|\left(g_{n}\right)_{\Delta}^{\prime}-\left(h_{m}\right)_{\Delta}^{\prime}\right|^{2} d S=\lim _{m \rightarrow \infty} \int_{\Delta}\left|\left(\left(f_{n} \circ \gamma\right)_{\Delta}-\left(h_{m}\right)_{\Delta}\right)^{\prime}\right|^{2} d S \\
& =4 \lim _{m \rightarrow \infty} \int_{\Delta}\left|\partial \operatorname{Re}\left(\left(f_{n} \circ \gamma\right)_{\Delta}-\left(h_{m}\right)_{\Delta}\right)\right|^{2} d S \\
& \leq 4 \lim _{m \rightarrow \infty} \int_{\Delta}\left|\partial \operatorname{Re}\left(\left(f_{n}\right)_{\Delta} \circ \varphi-\left(h_{m}\right)_{\Delta}\right)\right|^{2} d S=4 \int_{\Delta}\left|\partial \operatorname{Re}\left(\left(f_{n}\right)_{\Delta} \circ \varphi-g_{\Delta}\right)\right|^{2} d S \\
& =4 \int_{\Delta}\left|\partial \operatorname{Re}\left(\left(f_{n}\right)_{\Delta} \circ \varphi-f_{\Delta} \circ \varphi\right)\right|^{2} d S=4 \int_{\Delta}\left|\partial \operatorname{Re}\left(\left(f_{n}-f\right)_{\Delta} \circ \varphi\right)\right|^{2} d S
\end{aligned}
$$

where

$$
\partial=\frac{1}{2}\left(\frac{\partial}{\partial x}-i \frac{\partial}{\partial y}\right), \quad \bar{\partial}=\frac{1}{2}\left(\frac{\partial}{\partial x}+i \frac{\partial}{\partial y}\right)
$$

are formal derivatives. This and the $K$-quasi-invariance of the Dirichlet integral, cf. $[\mathrm{A}]$, lead to

$$
\begin{aligned}
4 \int_{\Delta}\left|\partial \operatorname{Re}\left(\left(f_{n}-f\right)_{\Delta} \circ \varphi\right)\right|^{2} d S & \leq 4 K(\gamma) \int_{\Delta}\left|\partial \operatorname{Re}\left(f_{n}-f\right)_{\Delta}\right|^{2} d S \\
& =K(\gamma) \int_{\Delta}\left|\left(f_{n}-f\right)_{\Delta}^{\prime}\right|^{2} d S \\
& =K(\gamma)\left\|f_{n}-f\right\|_{\mathbb{H}}^{2} \rightarrow 0 \quad \text { as } n \rightarrow \infty
\end{aligned}
$$


Hence by $(2.3)$

$$
\left\|B_{\gamma}\left(f_{n}\right)-g\right\|_{\mathbb{H}} \rightarrow 0 \quad \text { as } n \rightarrow \infty
$$

On the other hand, by the continuity of the operator $B_{\gamma}:\left(\mathbb{H},\|\cdot\|_{\mathbb{H}}\right) \rightarrow\left(\mathbb{H},\|\cdot\|_{\mathbb{H}}\right)$ and (2.2) we have

$$
\left\|B_{\gamma}\left(f_{n}\right)-B_{\gamma}(f)\right\|_{\mathbb{H}} \rightarrow 0 \quad \text { as } n \rightarrow \infty .
$$

This together with (2.4) yields $g=B_{\gamma}(f)$ which ends the proof.

The following main theorem involves the notion of a regular quasiconformal Teichmüller mapping. We recall that a quasiconformal self-mapping $\varphi$ of the unit disc $\Delta$ is said to be a regular Teichmüller mapping if there exists an analytic function $\psi: \Delta \rightarrow \mathbb{C}$ and a constant $k, 0 \leq k<1$, such that the complex dilatation of $\varphi$ has the form

$$
\frac{\bar{\partial} \varphi}{\partial \varphi}=k \frac{\bar{\psi}}{|\psi|} .
$$

THEOREM 2.2. If $f \in \mathbb{H},\|f\|_{\mathbb{H}}>0$, and $\varphi$ is a regular quasiconformal Teichmüller extension of an automorphism $\gamma \in \mathbb{Q}_{\mathbf{T}}$ to $\Delta$ with the complex dilatation

$$
\frac{\bar{\partial} \varphi}{\partial \varphi}=-\frac{1}{\lambda} \frac{\overline{f_{\Delta}^{\prime}}}{{f_{\Delta}^{\prime}}^{\prime}}
$$

where $\lambda,|\lambda|>1$, is a real constant then $\lambda \in \Lambda_{\gamma}^{*}$ and $|\lambda|$ is the smallest positive eigenvalue of $\gamma$, i.e.

$$
|\lambda|=\min \left\{|\mu|: \mu \in \Lambda_{\gamma}^{*}\right\}=\min \left\{|\mu|: \mu \in \Lambda_{\gamma}\right\} .
$$

Moreover,

$$
\left\|A_{\gamma}\right\|=K(\gamma)=\frac{|\lambda|+1}{|\lambda|-1}
$$

and

$$
(\lambda+1) A(f)=(\lambda-1) A_{\gamma}(f) .
$$

Proof. Let $G$ be a complex function in the unit disc $\Delta$ such that

$$
G \circ \varphi=\overline{f_{\Delta}}-\lambda f_{\Delta} .
$$

Differentiating both sides of this equality with respect to $z$ and $\bar{z}$ we get the simultaneous equations

$$
\begin{aligned}
& (\partial G) \circ \varphi \partial \varphi+(\bar{\partial} G) \circ \varphi \partial \bar{\varphi}=-\lambda f_{\Delta}^{\prime}, \\
& (\partial G) \circ \varphi \bar{\partial} \varphi+(\bar{\partial} G) \circ \varphi \bar{\partial} \bar{\varphi}=\bar{f}_{\Delta}^{\prime} .
\end{aligned}
$$

Since $\partial \varphi \bar{\partial} \bar{\varphi}-\bar{\partial} \varphi \partial \bar{\varphi}=\partial \varphi \overline{\partial \varphi}-\bar{\partial} \varphi \overline{\bar{\partial} \varphi}=|\partial \varphi|^{2}-|\bar{\partial} \varphi|^{2}>0$ a.e. in $\Delta$, (2.6) implies that $\bar{\partial} G=0$ a.e. in $\Delta$. This way $G$ is an analytic function in $\Delta$, cf. [A]. Moreover, by the quasi-invariance of the Dirichlet integral we derive from the equality (2.10) that 


$$
\begin{aligned}
\int_{\Delta}\left|G^{\prime}\right|^{2} d S & =4 \int_{\Delta}\left|\partial \operatorname{Re}\left(\left(\overline{f_{\Delta}}-\lambda f_{\Delta}\right) \circ \varphi^{-1}\right)\right|^{2} d S \\
& \leq 4 K\left(\varphi^{-1}\right)|1-\lambda|^{2} \int_{\Delta}\left|\partial \operatorname{Re} f_{\Delta}\right|^{2} d S=K(\varphi)|1-\lambda|^{2} \int_{\Delta}\left|f_{\Delta}^{\prime}\right|^{2} d S<\infty .
\end{aligned}
$$

Thus there exists a function $g \in \mathbb{H}$ such that $G(z)=g_{\Delta}(z)+G(0), z \in \Delta$, and by the equality (2.10) we get on $\Delta$

$$
\begin{aligned}
& \operatorname{Re} g_{\Delta} \circ \varphi+\operatorname{Re} G(0)=(1-\lambda) \operatorname{Re} f_{\Delta}, \\
& \operatorname{Im} g_{\Delta} \circ \varphi+\operatorname{Im} G(0)=-(1+\lambda) \operatorname{Im} f_{\Delta} .
\end{aligned}
$$

Hence, by the definition of the harmonic conjugation operator $A$ and Lemma 2.1 we obtain

$$
B_{\gamma}(g)=(1-\lambda) f \quad \text { and } \quad B_{\gamma}(A(g))=-(1+\lambda) A(f) .
$$

This gives by (1.1)

$$
(1+\lambda) A(f)=(\lambda-1) B_{\gamma} A B_{\gamma}^{-1}(f)=(\lambda-1) A_{\gamma}(f)
$$

which proves the equality (2.9). This means that $\lambda \in \Lambda_{\gamma}^{*}$. It follows from the assumption of Lemma 2.1 that $\varphi$ is a $K$-quasiconformal regular Teichmüller extension of the automorphism $\gamma$ on $\Delta$ with $K=(|\lambda|+1) /(|\lambda|-1)$. Thus

$$
\left\|A_{\gamma}\right\| \leq K(\gamma) \leq K
$$

If $\lambda<-1$ then by the property (vi) $|\lambda|=-\lambda \in \Lambda_{\gamma}^{*}$ as well. Therefore there exists $h \in \mathbb{H},\|h\|_{\mathbb{H}}=1$, satisfying

$$
(|\lambda|+1) A(h)=(|\lambda|-1) A_{\gamma}(h) .
$$

Hence

$$
\left\|A_{\gamma}(h)\right\|_{\mathbb{H}}=\left\|A A_{\gamma}(h)\right\|_{\mathbb{H}}=\frac{|\lambda|+1}{|\lambda|-1}=K
$$

because $A^{2}=-I$ and $A$ is an isometry of the space $\left(\mathbb{H},\|\cdot\|_{\mathbb{H}}\right)$ onto itself, cf. [P2, Theorem 1.3]. Thus $\left\|A_{\gamma}\right\| \geq K$. This together with (2.11) gives the equality (2.8) which yields the equality (2.7) because of the properties (ii) and (iii). This completes the proof.

Remark. This result seems to be closely related to that in [Kü, p. 302]. The equality (2.8) states additionally that the mapping $\varphi$ in Theorem 2.2 is the extremal quasiconformal extension of $\gamma$ to the unit disc $\Delta$, i.e. an extension with the smallest maximal dilatation. This way we have proved, by the way, Strebel's theorem, cf. [St1], [St2], [L], in the case when the function $\psi$ in (2.5) is a square of some analytic function, square integrable on $\Delta$.

The smallest positive eigenvalue of a quasisymmetric automorphism $\gamma$ of the unit circle plays a particularly important role among other eigenvalues of $\gamma$. Namely, every positive eigenvalue $\lambda \in \Lambda_{\gamma}^{*}$ can be expressed as the smallest positive 
eigenvalue of some other quasisymmetric automorphism $\sigma \in \mathbb{Q}_{\mathbf{T}}$. This interesting fact is the subject of the following corollary to Theorem 2.2.

COROllary 2.3. If $\lambda \in \Lambda_{\gamma}^{*}, \lambda>0$ is any eigenvalue of an automorphism $\gamma \in \mathbb{Q}_{\mathbf{T}}$ then there exists an automorphism $\sigma \in \mathbb{Q}_{\mathbf{T}}$ such that

$$
|\lambda|=\min \left\{|\mu|: \mu \in \Lambda_{\sigma}^{*}\right\}=\min \left\{|\mu|: \mu \in \Lambda_{\sigma}\right\}
$$

and

$$
K(\sigma)=\left\|A_{\sigma}\right\|=\frac{\lambda+1}{\lambda-1} .
$$

Moreover, the automorphism $\sigma \in \mathbb{Q}_{\mathbf{T}}(K)$ admits a $K(\sigma)$-quasiconformal extension $\varphi$ to the unit disc $\Delta$ with a complex dilatation

$$
\bar{\partial} \varphi=-\frac{1}{\lambda \varphi} \frac{\overline{f_{\Delta}^{\prime}}}{\overline{f_{\Delta}^{\prime}}}
$$

where

$$
(\lambda+1) A(f)=(\lambda-1) A_{\sigma}(f) .
$$

Proof. Assume $\lambda \in \Lambda_{\gamma}^{*}, \lambda>0$ is an eigenvalue of an automorphism $\gamma \in \mathbb{Q}_{\mathbf{T}}$. Then there exists a function $f \in \mathbb{H},\|f\|_{\mathbb{H}}=1$ satisfying (1.3). It follows from the mapping theorem, cf. [LV; p. 194], also [B1], [B2], [EK] that there exists a solution $\varphi$ of the Beltrami equation (2.14) being a $K$-quasiconformal self-mapping of $\Delta$ with $K=(\lambda+1) /(\lambda-1)$. It is well known that $\varphi$ has a continuous extension to $\mathbf{T}$ as a quasisymmetric automorphism $\sigma \in \mathbb{Q}_{\mathbf{T}}$, cf. [LV]. Then the assumptions of Theorem 2.2 are satisfied for the automorphism $\sigma \in \mathbb{Q}_{\mathbf{T}}$ which satisfies the equalities $(2.12),(2.13)$ and $(2.15)$. This ends the proof.

\section{References}

[A] L. V. Ahlfors, Lectures on Quasiconformal Mappings, D. Van Nostrand, Princeton, 1966.

[BS] S. Bergman and M. Schiffer, Kernel functions and conformal mapping, Compositio Math. 8 (1951), 205-249.

[B1] B. Bojarski, Homeomorphic solution of Beltrami systems, Dokl. Akad. Nauk SSSR 102 (1955), 661-664 (in Russian).

[B2] - Generalized solutions of a system of differential equations of the first order and elliptic type with discontinuous coefficients, Mat. Sb. N.S. 43 (1957), 451-503 (in Russian).

[G] J. B. Garnett, Bounded Analytic Functions, Academic Press, New York, 1981.

[K] J. G. Krzyż, Quasicircles and harmonic measure, Ann. Acad. Sci. Fenn. Ser. A I Math. 12 (1987), 19-24.

[KP] J. G. Krzyż and D. Partyka, Generalized Neumann-Poincaré operator, chord-arc curves and Fredholm eigenvalues, Complex Variables, Theory Appl. 21 (1993), 253263.

[Kü] R. Kühnau, Wann sind die Grunskyschen Koeffizientenbedingungen hinreichend für Q-quasikonforme Fortsetzbarkeit?, Comment. Math. Helv. 61 (1986), 290-307. 
[L] O. Lehto, Univalent Functions and Teichmüller Spaces, Graduate Texts in Math. 109 Springer, New York, 1987.

[LV] O. Lehto and K. I. Virtanen, Quasiconformal Mappings in the Plane, 2nd ed., Grundlehren Math. Wiss. 126, Springer, New York, 1973.

[ŁK] J. Ławrynowicz and J. G. Krzyż, Quasiconformal Mappings in the Plane: Parametrical Methods, Lecture Notes in Math. 978, Springer, Berlin, 1983.

[P1] D. Partyka, Spectral values of a quasicircle, Complex Variables Theory Appl., to appear.

[P2] _- Generalized harmonic conjugation operator, Proceedings of the Fourth FinnishPolish Summer School in Complex Analysis at Jyväskylä, Ber. Univ. Jyväskylä Math. Inst. 55 (1993), 143-155.

[P3] -, Spectral values and eigenvalues of a quasicircle, preprint.

[S] M. Schiffer, The Fredholm eigenvalues of plane domains, Pacific J. Math. 7 (1957), $1187-1225$.

[St1] K. Strebel, Zur Frage der Eindeutigkeit extremaler quasikonformer Abbildungen des Einheitskreises I, Comment. Math. Helv. 36 (1962), 306-323.

[St2] —, Zur Frage der Eindeutigkeit extremaler quasikonformer Abbildungen des Einheitskreises II, ibid. 39 (1964), 77-89. 